\title{
Resistência à tração de emendas dentadas de madeira de Manilkara huberi para o emprego em madeira laminada colada
}

\author{
Tensile strength of finger joints made of Manilkara \\ huberi wood for use in glued laminated timber
}

\section{Julio Cesar Molina \\ Carlito Calil Neto \\ André Luis Christoforo}

\section{Resumo}

A

utilização de elementos estruturais de madeira laminada (ou lamelada) colada (MLC) requer um estudo experimental das combinações entre a espécie de madeira e o adesivo utilizado. Este trabalho objetivou o estudo da combinação espécie-adesivo na resistência a tração e na falha da linha de cola de corpos de prova de madeira de maçaranduba (Manilkara huberi) confeccionados com e sem emendas dentadas finger joint. As colagens foram realizadas com lâminas sem tratamento preservativo, com aproximadamente $12 \%$ de umidade e com o emprego do adesivo mono-componente do tipo poliuretano Purbond HB 712. A gramatura de adesivo utilizada nas emendas dentadas foi linha simples de cola com aproximadamente $200 \mathrm{~g} \mathrm{~m}-2$ e pressão de contato igual a $10 \mathrm{MPa}$. Após sete dias de cura do adesivo foram realizados testes de tração nos corpos de prova com e sem emendas dentadas, possibilitando julgar, também por meio da análise de variância, a eficiência das emendas testadas. Os resultados da experimentação revelaram a ineficiência da interface entre o adesivo e a madeira de analisada, pois a ruptura ocorreu na linha de cola, categorizando modo de ruptura do tipo 1 , que corresponde à pior situação de falha.

Palavras-chaves: Juntas dentadas coladas. Madeira de maçaranduba. Adesivo.

Julio Cesar Molina Universidade Estadual Paulista Itapeva - SP - Brasil

Carlito Calil Neto Universidade de São Paulo São Carlos - SP - Brasil

André Luis Christoforo Universidade Federal de São Carlos São Carlos - SP - Brasil

Recebido em 22/01/14 Aceito em 07/09/15

\section{Abstract}

The use of glued laminated timber structural elements requires an experimental study of combinations of wood species and the adhesives used. This aim of this study was to investigate the wood species-adhesive combination in the tensile strength and failure modes of Manilkara huberi wood species manufactured with and without finger joints. The assemblies were made with untreated layers of wood with approximately $12 \%$ moisture using the polyurethane-based mono-component adhesive model Purbond HB 712. The amount of adhesive used in the finger joint was a single line of glue with $200 \mathrm{~g} \mathrm{m-2}$ and pressure of $10 \mathrm{MPa}$. After 07 days of curing the adhesive, tensile tests were performed on specimens with and without finger joints, allowing the assessment of the efficiency of the joints also through variance analysis. The results of the tests revealed the ineffectiveness of the interface between the adhesive and the Manilkara huberi wood, since the rupture occurred on the glue line, configuring a type 1 failure mode, representing the worst rupture situation.

Keywords: Finger joint. Manilkara huberi wood. Adhesive. 


\section{Introdução}

A madeira laminada colada (MLC), ou madeira lamelada colada, é um produto engenhado que consiste em uma das formas de utilização da madeira, preferencialmente de reflorestamento, que há vários anos vem sendo estudada no Brasil. Normalmente, as madeiras mais adequadas para o emprego em MLC são as de reflorestamento como as coníferas e algumas dicotiledôneas (folhosas). As espécies de coníferas mais utilizadas são os pínus, e entre as dicotiledôneas, os eucaliptos (CALIL NETO, 2008).

A MLC é formada basicamente por tábuas de madeira unidas longitudinalmente e depois coladas umas sobre as outras. Além disso, na produção de elementos de MLC são necessárias lâminas grandes, as quais são obtidas por emendas dentadas. Vale mencionar que a geometria dos elementos estruturais de MLC, principalmente nas regiões das conexões, eleva o estado de tensões a níveis máximos, conduzindo a falhas localizadas dos materiais para valores de carga de serviço (FIORELLI; DIAS, 2006; SEGUNDINHO et al., 2013), e que para algumas espécies de madeira, como é o caso das folhosas, as tiloses, que se originam em função de processos fisiológicos naturais, podem diminuir a permeabilidade, prejudicando a secagem da madeira (CALIL NETO, 2011), e uma boa interface com os adesivos utilizados.

Macedo (1996) estudou emendas dentadas em madeiras de Pinus elliottii e Eucalyptus grandis coladas com adesivo sintético à base de resorcinol formol, comercializado como Cascophen RS-216, que obtiveram bons resultados de resistência à tração na direção das fibras. Macedo e Calil Neto (2007) e Segundinho et al. (2013) afirmam que a qualidade das emendas depende diretamente da espécie de madeira, do teor de umidade e do tipo de adesivo utilizado.

Segundo Zangiácomo e Lahr (2007), madeiras tropicais, como o cedrinho (Erisma sp.), coladas com adesivos do tipo Cascophen e também com adesivos à base de mamona podem ser utilizadas na produção de MLC.

A MLC requer precisão de fabricação em todos os seus estágios (FIORELLI; DIAS, 2006). É necessário que haja um controle de qualidade na produção para assegurar que as propriedades da MLC sejam adequadas com as resistências especificadas de projeto (MACEDO; CALIL NETO, 2007). O controle de qualidade de MLC, neste caso, é com base na Canadian Standards Association (CANADIAN..., 2006). Ainda nesse contexto, o sistema de controle de qualidade é definido pelas ações realizadas por um fabricante em relação aos materiais, métodos, equipamentos, mão de obra e produto final, para satisfazer os requisitos necessários de uma norma de controle de qualidade como aquela proposta pelo American Institute of Timber Construction (AMERICAN INSTITUTE..., 2005).

O programa de qualidade conta com vários especialistas, que entendem da importância do processo de certificação para conseguir a confiança do consumidor e a qualidade final do produto. Para predizer o desempenho estrutural da MLC com base nesse programa é necessária a realização de ensaios mecânicos diários, sistemas de avaliação em pontos estratégicos da produção e inspeção constante durante o processo de produção (CALIL NETO, 2011).

Destaca-se que todas essas atividades devem ser auditadas e verificadas por inspetores credenciados. É possível colar praticamente todos os tipos de madeiras. Entretanto, algumas espécies possuem características físicas e químicas que exigem o emprego de colas especiais ou a modificação destas, que são normalmente comercializadas para uso em madeiras estruturais (CALIL NETO, 2008).

Por outro lado, como o sistema de MLC é ainda pouco utilizado no Brasil, é evidente que estudos devam ser realizados no sentido de se proceder em cada região ou estado a uma investigação da interação da relação espécie de madeira-adesivo para uma caracterização das madeiras que melhor possam se adaptar a essa técnica de colagem. Deve ter destaque, principalmente, a adequabilidade de madeiras provenientes de florestas plantadas, como, por exemplo, as espécies de pínus e eucaliptos ao referido sistema de emenda (CALIL NETO, 2011).

$\mathrm{Na}$ maioria dos casos a escolha da cola, entre caseína, resorcina ou ureia-formol, e mais recentemente a melamina, depende mais das condições de uso da estrutura do que do tipo da madeira considerada. Logo, é preciso levar em consideração, principalmente, o meio a que a estrutura vai estar submetida, para controle da temperatura e teor de umidade (SELLERS JÚNIOR, 2001).

A madeira de maçaranduba (Manilkara huberi) é proveniente da região amazônica. Trata-se de uma árvore com cerca de 40 a $50 \mathrm{~m}$ de altura que é encontrada geralmente nas regiões de terra firme a até $700 \mathrm{~m}$ de altitude. Entre as espécies do gênero, a Manilkara huberi é a mais conhecida e com a 
maior distribuição na Amazônia (INSTITUTO..., 1983).

Apesar de ser facilmente reconhecida por apresentar folhas grandes e amarelas na face abaxial, é confundida com outras espécies do gênero devido à similaridade de seus troncos. A espécie do gênero é mais valorizada pela qualidade de sua madeira, que é muito pesada e resistente, com densidade aparente e resistência à compressão na direção das fibras aproximadas de $1.143 \mathrm{~kg} \mathrm{~m}^{-3}$ e 82,9 MPa respectivamente. É usada principalmente em construções externas, como dormentes e pisos industriais.

Vale mencionar que espécies com menores densidades (como os pínus, que apresentam valores de densidade aparente em torno $600 \mathrm{~kg} \mathrm{~m}^{-3}$ e resistência média à compressão paralela às fibras de $40 \mathrm{MPa}$ ) têm maior potencial para MLC por aderirem melhor durante a colagem.

O objetivo principal deste trabalho foi estudar a combinação espécie-adesivo na resistência à tração e na falha da linha de cola de corpos de prova de madeira de maçaranduba (Manilkara huberi) confeccionados com e sem emendas dentadas do tipo finger joint.

\section{Material e métodos}

Para este estudo foram confeccionados 6 corpos de prova de tração para os testes envolvendo emendas dentadas. Nesse caso as dimensões dos corpos de prova foram $130 \mathrm{~mm} \times 50 \mathrm{~mm} \times 20 \mathrm{~mm}$ (comprimento $\mathrm{x}$ largura $\mathrm{x}$ espessura). Foram também confeccionados outros 6 corpos de prova de madeira com as mesmas dimensões, porém sem as emendas dentadas, os quais foram usados como referência para comparação dos valores de resistência última na tração $\left(f_{U}\right)$, para subsidiar a interpretação dos resultados.

Cabe ressaltar que o número de amostras ensaiadas assim como as dimensões dos corpos de prova para os ensaios de tração com e sem emendas dentadas foram definidos em função das dimensões e da disponibilidade das peças de madeira de maçaranduba fornecidas por um fabricante de emendas dentadas de Manaus, AM.

A análise do comportamento das emendas dentadas (modos de ruptura) foi efetuada com base na American Society for Testing and Materials (AMERICAN SOCIETY..., 2005).

Os ensaios de tração, conforme detalhes apresentados na Figura 1, foram realizados de acordo com as recomendações da Norma Brasileira Regulamentadora NBR 7190, da Associação Brasileira de Normas Técnicas (ABNT, 1997).

Os ensaios foram realizados nas dependências do Laboratório de Madeiras e de Estruturas de Madeiras (LaMEM) da Escola de Engenharia de São Carlos (EESC), da Universidade de São Paulo (USP).

Cada corpo de prova foi composto de uma emenda dentada horizontal (padrão americano) posicionada no meio do corpo de prova, sendo o tamanho do dente igual a $15 \mathrm{~mm}$ de comprimento (Figura 2). Essas emendas foram confeccionadas tendo em vista a disponibilidade do equipamento para a fabricação delas.

A confecção dos corpos de prova para os ensaios das ligações dentadas foi realizada com lamelas sem tratamento preservativo, com umidade de $12 \%$ $\pm 1 \%$. Foi utilizado na colagem das emendas dentadas o adesivo estrutural monocomponente Purbond HB 712, à base de poliuretano. A gramatura de adesivo utilizada nas emendas dentadas foi aquela especificada pelo fabricante, ou seja, linha simples de cola com 180 a $200 \mathrm{~g} \mathrm{~m}^{-2}$. A pressão utilizada na colagem foi de $10 \mathrm{MPa}$. Na Figura 3 são indicados os principais detalhes da emenda dentada, assim como da colagem delas.

\section{Figura 1 - Ensaio de tração para avaliação da resistência da emenda dentada}

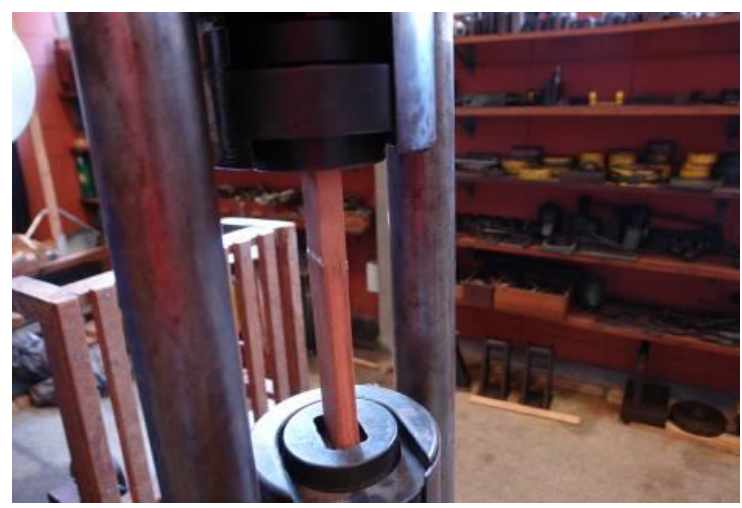


Figura 2 - Detalhe da emenda dentada

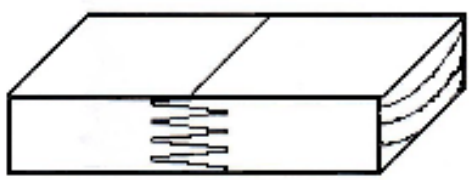

(a) Posição horizontal

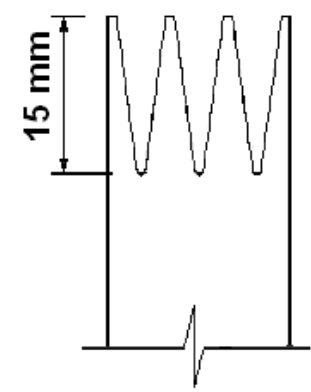

(b) Tamanho do dente

Figura 3 - Corpos de prova de tração para ensaio das emendas dentadas

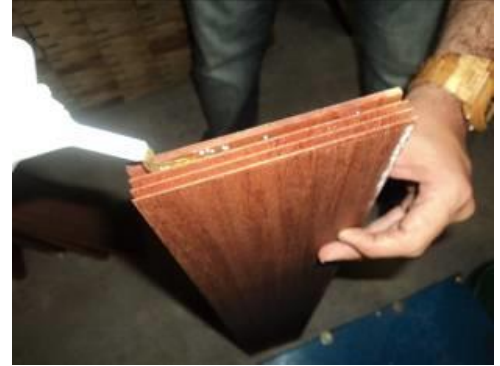

(a) Aplicação do adesivo

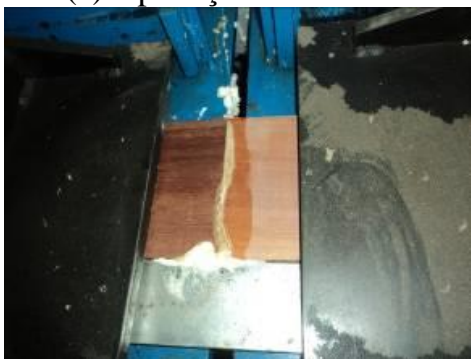

(c) Colagem

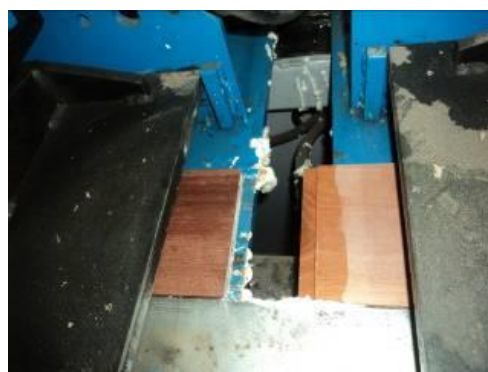

(b) Posicionamento na prensa

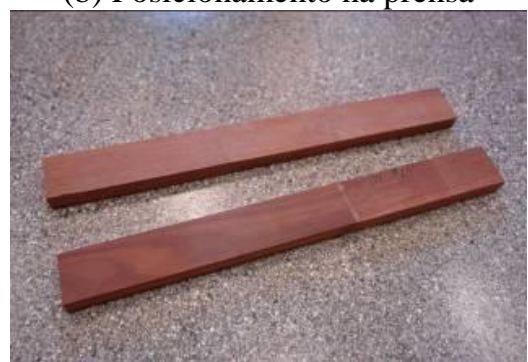

(d) Corpos de prova prontos

emendas dentadas apresentaram ruptura do tipo frágil. A ruptura ocorreu na linha de cola, nesse caso, assim como ilustra a Figura 4.

Os valores de resistência das ligações dentadas assim como os modos de ruptura obtidos a partir dos ensaios de tração dos corpos de prova são apresentados na Tabela 1.

Os resultados do teste de normalidade e de homogeneidade de variâncias são ilustrados na Figura 5. Uma vez que os P-valores encontrados foram superiores à significância dos testes, constata-se que as distribuições são normais e que as médias entre os tratamentos são equivalentes, validando o modelo da ANOVA.

Na Tabela 2 estão apresentados os resultados da ANOVA do fator emenda dentada (com e sem emenda) nos valores de resistência à tração dos corpos de prova testados.

Pelo fato de P-valor encontrado na Tabela 2 ser inferior a 0,05 , constata-se a não equivalência dos

224 Molina, J. C.; Calil NETO, C.; Christoforo, A. L. 
resultados de resistência última na tração - $f_{U}$, evidenciando que os valores de resistência na tração dos corpos de prova com emenda foram significativamente inferiores $(26 \%)$ aos valores de resistência da madeira de maçaranduba sem emenda. Embora o resíduo, apresentado na Tabela

Figura 4 - Ensaio de tração

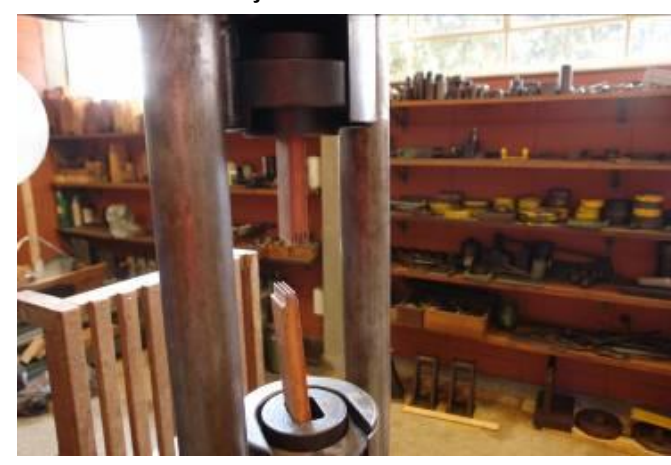

(a)Ruptura do tipo frágil
2, seja pequeno em função da quantidade de corpos de prova utilizados, a mesma está em conformidade com o que propõe a NBR 7190 (ABNT, 1997) para uma análise simplificada de espécies de madeira conhecidas.

Tabela 1 - Módulos de ruptura na tração paralela às fibras da madeira de maçaranduba sem emendas e das emendas dentadas

\begin{tabular}{c|c|c}
\hline \multirow{2}{*}{ Módulo de Ruptura } & Emendas Dentadas & Madeira sem Emenda \\
\cline { 2 - 3 } & $\boldsymbol{f}_{U}(\mathbf{M P a})$ & $\boldsymbol{f}_{\boldsymbol{U}}(\mathbf{M P a})$ \\
\hline Media & 17,06 & 23,12 \\
Coeficiente de Variação $(\%)$ & $26 \%$ & $19 \%$ \\
\hline
\end{tabular}

Nota: $f_{U}=$ resistência última na tração.

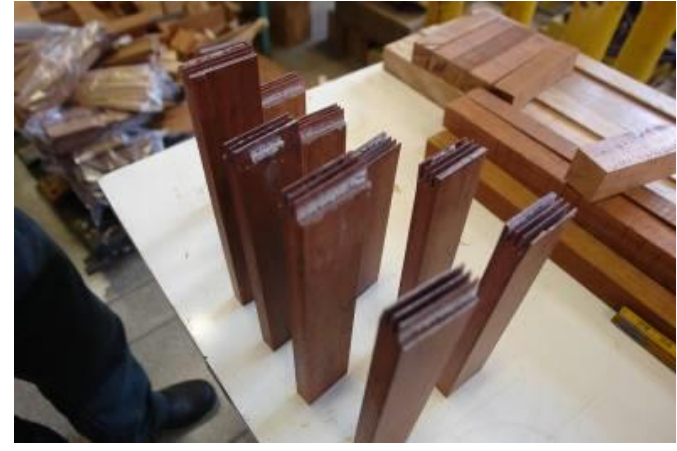

(b) Ruptura na linha de cola

Figura 5 - Resultados dos testes de normalidade

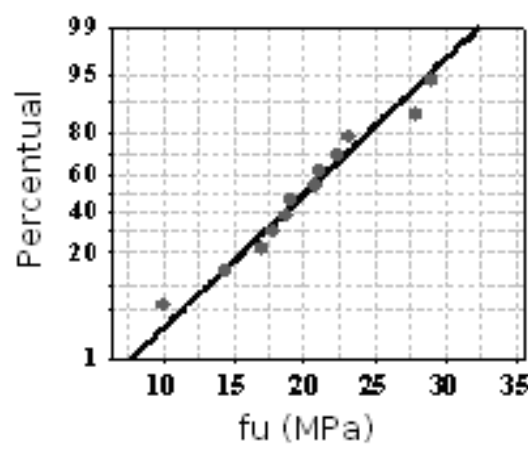

(a) Homogeneidade de variâncias

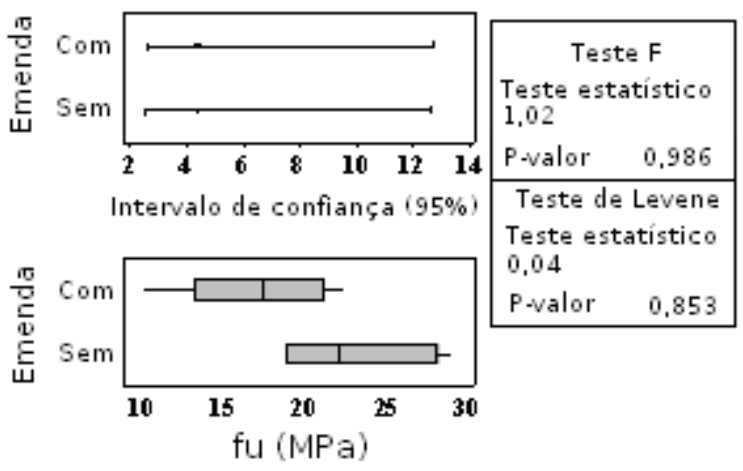

(b) Valores de resistência na tração

Nota: Legenda:

Tabela 2 - ANOVA do fator emenda dentada (com e sem) nos valores de resistência na tração

\begin{tabular}{cccccc}
\hline Fonte & GL & SQ & MQ & F & P-valor \\
\hline Emenda & 1 & 110,4 & 110,4 & 5,61 & 0,390 \\
Resíduo & 10 & 196,8 & 19,7 & & \\
\hline Total & 11 & 307,2 & & & \\
\hline
\end{tabular}

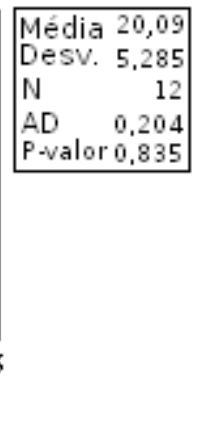

AD $\quad 0.20$

\begin{tabular}{|c|c|c|}
\hline 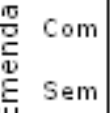 & $\longrightarrow \square$ & $\square$ \\
\hline & $\begin{array}{l}10 \quad 15 \\
\text { fu } \\
\text { (b) Valore }\end{array}$ & $\begin{array}{l}\mathbf{2 0} \mathbf{2 5} \\
(\mathrm{MPa}) \\
\text { s de resistêr }\end{array}$ \\
\hline MQ & $\mathbf{F}$ & P-valor \\
\hline $\begin{array}{c}110,4 \\
19,7\end{array}$ & 5,61 & 0,390 \\
\hline
\end{tabular}

$\mathrm{GL}=$ graus de liberdade;

$\mathrm{SQ}=$ soma dos quadrados dos resíduos; e

$M Q=$ média dos quadrados dos resíduos. 
Quando se verifica o comportamento de ligações dentadas, espera-se que a ruptura da região da ligação ocorra nos dentes da emenda de madeira, em vez de na linha de cola (SELLERS JÚNIOR, 2001). Além disso, a maioria dos trabalhos dessa natureza se refere a madeiras de pínus com diferentes tipos de adesivos, impedindo, muitas vezes, o estabelecimento de conclusões gerais (CALIL NETO, 2008).

Nos ensaios de tração das emendas dos corpos de prova efetuados neste trabalho observou-se que a ruptura da ligação dentada ocorreu na linha de cola. Esse tipo de ruptura é entendido, segundo a ASTM D - 4688 (AMERICAN SOCIETY..., 2005), como ruptura do tipo 1 , que corresponde à pior situação de ruptura. Constata-se, assim, que a colagem, neste caso, não foi suficiente para garantir a qualidade da ligação. A madeira de maçaranduba apresentou resistência média na tração de 23,12 MPa e baixa penetração ao adesivo utilizado nas regiões das ligações dentadas. Essa baixa penetração do adesivo pode ser decorrente da presença de tiloses na madeira analisada, o que dificulta a penetração do adesivo. No entanto, nesse caso, cabe uma verificação mais detalhada com o auxílio de microscopia óptica.

\section{Conclusões}

A combinação entre a espécie de maçaranduba e o adesivo monocomponente à base de adesivo poliuretano Purbond HB obteve resultados insatisfatórios para o uso estrutural. No entanto, para outras aplicações, como móveis e assoalhos, essa mesma combinação espécie-adesivo deve ser analisada de maneira específica.

Há a necessidade da realização de ensaios adicionais de delaminação e também de deformação lenta para a combinação espécieadesivo utilizada neste trabalho, de modo a se terem outros parâmetros de avaliação para a classificação ou desclassificação desse conjunto na fabricação de MLC estrutural.

Os corpos de prova com emendas dentadas apresentaram valores de resistência à tração inferiores aos corpos de prova ensaiados sem a presença da emenda dentada, evidenciando, assim como encontrado nos modos de ruptura, a pequena afinidade da resina poliuretana utilizada com a madeira de maçaranduba testada.

\section{Referências}

\section{AMERICAN INSTITUTE OF TIMBER}

CONSTRUCTION. AITC 115: standard for

fabricated structural glued laminated timber components and assemblies. Colorado, 2005.
AMERICAN SOCIETY OF TESTING AND MATERIALS. ASTM D 4688: standard test method for evaluating structural adhesives for finger jointing lumber. West Conshohocken, 2005.

ASSOCIAÇÃO BRASILEIRA DE NORMAS TÉCNICAS. NBR 7190: projeto de estruturas de madeira. Rio de Janeiro, 1997.

CALIL NETO, C. Recomendações Para o Controle de Qualidade na Produção de Madeira Laminada Colada (MLC) Certificada. 86 f. Itapeva, 2008. Monografia (Graduação Engenharia Industrial Madeireira) - Escola de Engenharia, Universidade Estadual Paulista "Júlio de Mesquita Filho", Itapeva, 2008.

\section{CALIL NETO, C. Madeira Laminada Colada}

(MLC): controle de qualidade em combinações espécie-adesivo -tratamento preservativo. São Carlos, 2011. 123 f. Dissertação (Mestrado em Ciência e Engenharia de Materiais) - Escola de Engenharia, Universidade de São Paulo, São Carlos, 2011.

\section{CANADIAN STANDARD ASSOCIATION.} CAN/CSA 0177 M1989: qualification code for manufacturers of structural glued-laminated timber. Mississauga, 2006.

FIORELLI, J.; DIAS, A. A. Fiberglass-Reinforced Glulam Beams: mechanical properties and theoretical model. Materials Research, São Carlos, v. 9, n. 3, p. 263-269, 2006.

\section{INSTITUTO DE PESQUISAS}

\section{TECNOLÓGICAS. Informações Sobre}

Madeiras: maçaranduba. 1983. Disponível em: http://www.ipt.br/informacoes_madeiras/4.htm. Acesso em: 10 jan. 2014.

MACEDO, A. N. Estudo de Emendas Dentadas em Madeira Laminada Colada (MLC): avaliação de método de ensaio. 115 f. São Carlos, 1996. Dissertação (Mestrado em Engenharia de Estruturas) - Escola de Engenharia, Universidade de São Paulo, São Carlos, 1996.

MACEDO, A. N.; CALIL NETO, C. Fadiga em Emendas Dentadas em Madeira Laminada Colada. Cadernos de Engenharia de Estruturas, São Carlos, v. 9, n. 37, p. 95-126, 2007.

SEGUNDINHO, P. G. A. et al. Avaliação de Vigas de Madeira Laminada Colada de Cedrinho (Erisma uncinatum Warm.). Cerne, Lavras, v. 19, n. 3, p. 441-449, 2013.

SELLERS JUNIOR, T. Wood Adhesive Innovation and Applications in North America. Forest Products Journal, Madison, v. 51, n. 6, p. 12-22, 2001. 
ZANGIÁCOMO, A. L.; LAHR, F. R. Emprego de Espécies Tropicais Alternativas na Produção de Elementos Estruturais de Madeira Laminada Colada. Caderno de Engenharia de Estruturas, São Carlos, v. 9, n. 40, p. 103-131, 2007.

\section{Agradecimentos}

Os autores agradecem a Coordenação de Aperfeiçoamento de Pessoal de Ensino Superior (Capes), pelo financiamento de parte desta pesquisa, e a Portela Indústria e Comércio de Madeiras Ltda., pelo fornecimento dos materiais para a pesquisa.

\section{Julio Cesar Molina}

Universidade Estadual Paulista | Rua Geraldo Alckimin, 519, Nossa Senhora de Fátima | Itapeva - SP - Brasil | CEP 13560-970 | Tel.: (15) 3524-9100 | E-mail.: molina@itapeva.unesp.br

\section{Carlito Calil Neto}

Departamento de Estruturas, Escola de Engenharia de São Carlos | Universidade de São Paulo | Av. Trabalhador Saocarlense, 400, Centro | São Carlos - SP - Brasil | Caixa Postal 359 | CEP 13566-590 | Tel.: (16) 3373-9320 | E-mail: netousp@gmail.com

\section{André Luis Christoforo}

Departamento de Engenharia Civil | Universidade Federal de São Carlos | Rodovia Washington Luís, km 235, SP310 | São Carlos - SP -

Brasil | CEP 36307-352 | Tel.: (16) 3351-8262 | E-mail: alchristoforo@yahoo.com.br

\section{Revista Ambiente Construído}

Associação Nacional de Tecnologia do Ambiente Construído

Av. Osvaldo Aranha, $99-3^{\circ}$ andar, Centro

Porto Alegre - RS - Brasil

CEP $90035-190$

Telefone: +55 (51) 3308-4084

Fax: +55 (51) 3308-4054

www.seer.ufrgs.br/ambienteconstruido

E-mail: ambienteconstruido@ufrgs.br 